\title{
Getting confidence for flying additive manufactured hardware
}

\author{
Fernando Lasagni $^{1} \cdot$ Jorge Vilanova $^{2} \cdot$ Antonio Periñán $^{1} \cdot$ Amadís Zorrilla $^{1} \cdot$ \\ Santos Tudela ${ }^{1} \cdot$ Vicente Gómez-Molinero $^{2}$
}

Received: 30 January 2016/Accepted: 15 June 2016/Published online: 23 June 2016

(c) Springer International Publishing Switzerland 2016

\begin{abstract}
In the next coming years, the European space industry must assume the challenge of implementing in an efficient way additive manufacturing (AM) technology due to the continuous need for reducing costs and improving performances. Together with the advantage of producing very complex and optimized geometries, AM can reduce drastically part weight and lead time of unique products (Tomlin and Meyer in Topology optimization of an additive layer manufactured (ALM) Aerospace part. In: The 7th Altair CAE Technology Conference, pp 1-9, 2011; Emmelmann et al. in Phys Procedia 12A:364-368, 2011). Similar to what happened during the implementation of composite materials within the aeronautics and space sectors at the early $80 \mathrm{~s}$, efforts must be directed to the development of fabrication and design rules, together with appropriate means of verification (Lasagni et al. in On the investigation of processing parameters and NDT inspection on additive manufacturing materials for future launchers, workshop on additive manufacturing for space applications. ESA-ESTEC, Noordwijk, The Netherlands, 2014). This work reports on the development, optimization, and AM fabrication of flight hardware for launchers, under the European Space Agency Future Launcher Preparation Programme. Articles for the clamp band separation system
\end{abstract}

Fernando Lasagni

flasagni@catec.aero

Jorge Vilanova

jorge.vilanova@airbus.com

1 CATEC, Center for Advanced Aerospace Technologies, C/Wilbur y Orville Wright 19, 41903 La Rinconada (Seville), Spain

2 Airbus Defence and Space, Space Systems, Avda. De Aragón 404, 28022 Madrid, Spain from Ariane 5 has been analysed together with other secondary structural items. The paper presents the full complete story, starting with the optimization of processing parameters for selective laser melting in different alloys, up to the qualification test of the whole system. A feasibility analysis for non-destructive evaluation is presented as well.

Keywords Additive manufacturing - Space applications . Launchers · Qualification

\section{Introduction}

The space industry is one of the most promising industrial sectors for the implementation of additive manufacturing technology. The combination of different issues, such as (1) the fabrication of unique products, (2) short series, (3) necessity for weight reduction through topology optimization, (4) structural components but not subjected to fatigue loads, etc., makes AM processes attractive for developing a large family of metallic parts for launcher and satellite applications.

One of the main goals of this work consists in acquiring the knowhow for answering two basic but relevant questions:

- The first one, whether AM is a sufficient mature technology to be ready for its use as space flight hardware in the framework of the Future Launcher Preparation Programme;

- In addition, in the second term, if AM technology can be used for reducing the product development cycle, and consequently development, production, and operational costs.

Weight reduction increasing the strength-to-weight is of paramount importance in the space industry, since 
components are usually expensive due to the high waste/long machining times of the conventional processes [4]. For that reason, a screening of launcher elements potentially candidates for fabrication in AM technologies has been carried out. The study includes the identification of critical areas, design, and optimization paths, and with the following goals:

1. Identify potential target applications in a Launch Vehicle system.

2. Assess technology readiness level (TRL)/industrial readiness level (IRL) of the proposed AM technologies for the relevant target applications.

3. Obtain procurement guidelines at materials and process level.

It is necessary to remark that most of the AM technologies, processes, materials, or components under development cannot be well linked directly to a specific TRL. The last is at a different degree of maturity depending on the manufacturing process, the type of application or required material. A technology or process can be mature for a specific part or structure, with specific dimensions and/or requirements, but it could be not available by far for other application, such as a component with similar geometry but different sizings, tolerances, finishing requirements, etc.

During this study, a preliminary analysis was performed considering seven different types of AM technologies (according to ASTM [5]), 27 AM processes, and all commercial available metallic materials [6]. Once discarded the less suitable, a list of issues has been studied in detail for analysing their potential in the short-term applications for the current launcher's market.

To accomplish the defined statement for identifying the TRL level of the selected AM process, a Technology and Verification Development Plan (TVDP) has been performed. A first Research and Development (R\&D) campaign has been completed, owing to reach TRL 3, including laboratory-based studies to physically validate the predictions, and covering relevant necessary information for launchers' applications.

First, test specimens and breadboard selection were derived from the launcher structural parts tradeoff. A definition of the design requirements has also been performed, including mechanical and physical properties (tensile strength, stiffness-to-weight ratio, fracture toughness, and thermal expansion).

In a second stage, a selection of manufacturing process and testing facilities has been performed based on the trade-off analysis. Finally, the TVDP process logic has been elaborated for test specimens and breadboard manufacturing, inspection, and verification process. The last was divided into two main stages of research: system setup and manufacturing parameters investigation, which was performed by means of the characterization of a series of samples for microstructural, mechanical in different alloys systems, and launcher vehicle breadboard demonstrators. These components have been manufactured in the targeted alloys to perform a preliminary study about the feasibility of the selected technology (Fig. 1).

Finally, a post-process phase was carried out. Quality control and verification of the breadboard demonstrators were performed, covering the NDE selection method, execution and feasibility analysis, evaluation of inner structure (flaws), and surface finish and dimensioning.

The background acquired during the development of test specimens and demonstrators was later applied in the next phase for the breadboard manufacturing. As parallel activity, a complete analysis for risk evaluation has been performed, together with the development of the associated fall-back solutions.

After the TVDP execution, a TRL assessment has been performed to answer the key questions and supporting the required evidence for a rigorous TRL maturation analysis.

\section{Technology and verification development plan}

\subsection{Technology development inception}

A detailed tradeoff has been performed for the different launcher stages, selecting the potential case studies accomplishing selection criteria, such as reduction of manufacturing costs, improved designs (allowing the implementation of new features which are not possible by traditional methods), between others. The main goal of the study was to increase the maturation of AM technology in the launchers field. For that, it was necessary to develop flight components to verify their real applicability and manufacturing feasibility, evaluating their potential benefits, limitations, and drawbacks. The selection of the breadboard components and manufacturing methods was based in the exploration of several alternatives, applying different criteria of materials, functionality, and complexity.

The selected AM technologies for the potential applications were finally Selective Laser Melting (SLM) and Electron Beam Melting (EBM), both Powder Bed Fusion (PBF) methods. Regarding the materials, three alloys with different possibilities for their application in space structures were selected: TiAI6V4, AISI 316L, and AlSi12.

Three components with a different functionality and shape were selected: a guide of a clamp band payload/ launcher separation system, a tripod bracket of the AR5 (Ariane5) equipment bay, and an umbilical connector 
Fig. 1 TVDP process logic

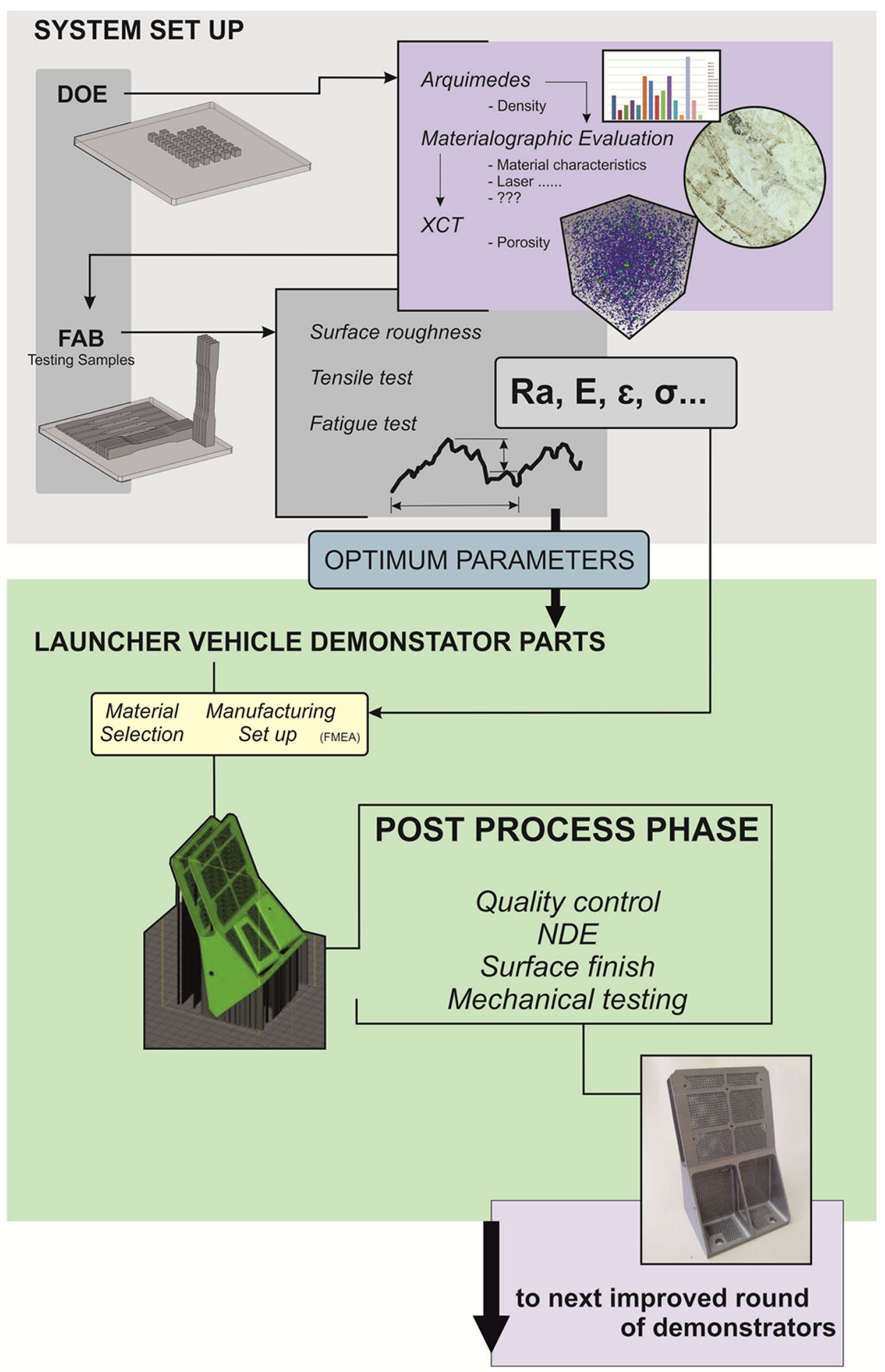

support of a payload adaptor (Fig. 2). In this way, a deep analysis has been carried out for the potential limitations in terms of geometries, size, and performance, and related to the selected alloy systems.

For the case of the flexible guide mechanism, the design was quite similar to the flight hardware. In the tripod bracket, some relevant design enhancements but without global shape modifications were introduced. Finally, for the case of the connector support, the design was completely disrupted with a topology and shape optimization, resulting in a component much different from the original one, but with same functionality and interfaces. 
(a)

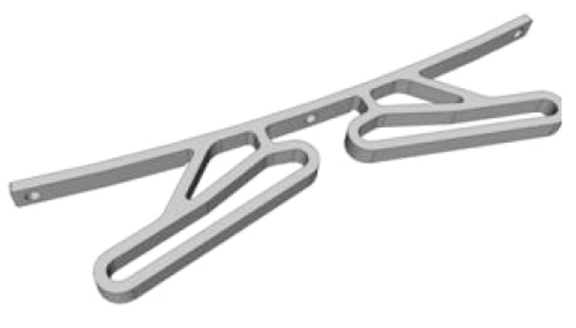

(b)

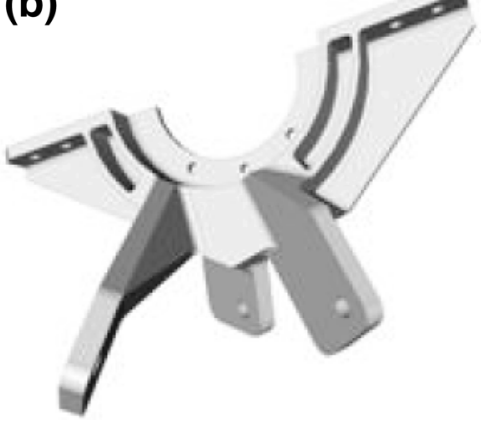

(c)

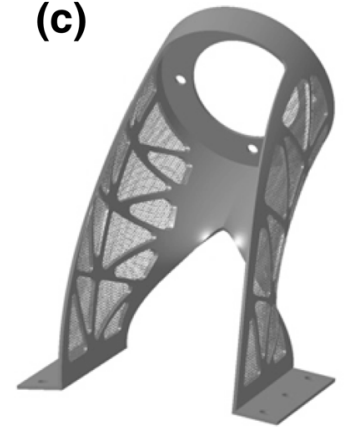

Fig. 2 Selected launch vehicle demonstrator parts: a flexible guide mechanism separation system in TiAl6V4; b tripod bracket AR5 EPS-S in Stainless Steel AISI 316L; and c connector support base AR5 VEGA AVUM in AlSi12

\subsection{Test report for specimens and demonstrators}

A laboratory-based study for the initiation of the TRL achievement plan has been performed to accomplish the specific targets stated in the TVDP. The activities were executed covering two main studies: (1) system setup, including manufacturing parameters investigation, and (2) launchers' vehicle breadboard demonstrators.

The SLM technology manufacturing parameters have been investigated for the three different alloys (AISI 316L, TiAl6V4, and AlSi12) through a series of planned design of experiments (DoE) [7]. Different samples with different manufacturing parameters were analysed for micro-structural characterization and void content determination.

Optimal manufacturing parameters were also established in terms of densification, surface quality, and mechanical behaviour of the alloy systems. Mechanical and surface characterization was executed on different building directions, and the initial information about technology performance was determined.

The design of experiments/DoE optimization was made with the goal of obtaining high-dense materials with a global void content below $1 \%$. Typical sphericity of voids was found to be about 0.5 (see [8] for definition). No cracks or platelet-like voids (with sphericity values larger than 0.15 ) have been observed, with more than $95 \%$ of the voids ranging below $0.18 \times 10^{-3} \mathrm{~mm}^{3}$. The quantification of the void content was executed by different methods: Archimedes immersion, for fast evaluation and tendency estimation of processing parameters, metallographic evaluation, for accurate characterization of microstructure, porous size, and porous volume characterization as well as laser pattern analysis, and finally, X-ray-computed tomographic (CT) analysis, using different building directions $\left(0^{\circ}\right.$ and $\left.45^{\circ}\right)$, for $3 \mathrm{D}$ the distribution of pores and quantification.

The mechanical tests performed using high-density-optimized parameters were the following (see Fig. 3 for sample visualization):
1. Vickers hardness measurements Measures have been taken in both parallel ( $z$ direction) and perpendicular ( $x y$ direction) to the building direction, according to E92-82 (standard test method for Vickers hardness of metallic materials). Results were also employed for the comparison and assessment of other mechanical properties, such as ultimate tensile strength and yield strength.

2. Tensile tests According to ECSS, Q-ST-70-45C and ASTM E8-E8M were performed on the investigated alloys, using specimens manufactured in different building directions $(x y z 90, x y 90 z$, and $x y z 0)$. The tensile test was employed for the evaluation of mechanical performance. The measured parameters were Young's modulus, yield and ultimate tensile strength, and elongation at break.

3. Compression test With different building orientations $\left(0^{\circ}, 90^{\circ}\right.$, and $\left.45^{\circ}\right)$, this testing methodology was carried out according to ECSS Q-ST-70-45C and ASTM E9-89a standards. These sets of test were performed to investigate the mechanical behaviour of the alloys according to the defined building orientation. The main parameter recorded on this test was the yield strength.

4. Fatigue testing, reporting on force controlled constant amplitude axial fatigue test results of specimens (for SS316L and Ti64) manufactured at XYZ90 building direction and developed on the previous stage. Testing methodology was carried out according to ECSS Q-ST-70-45C and ASTM E 466 standards. The number of failure cycles at different stress levels was investigated, too.

After these tests, an engineering analysis with three different methods for surface modification was studied: sandblasting, electro polishing, and electroplating. Parts and test specimens were fabricated using high-density-optimized parameters from DoE. Finally, sand blasting post 


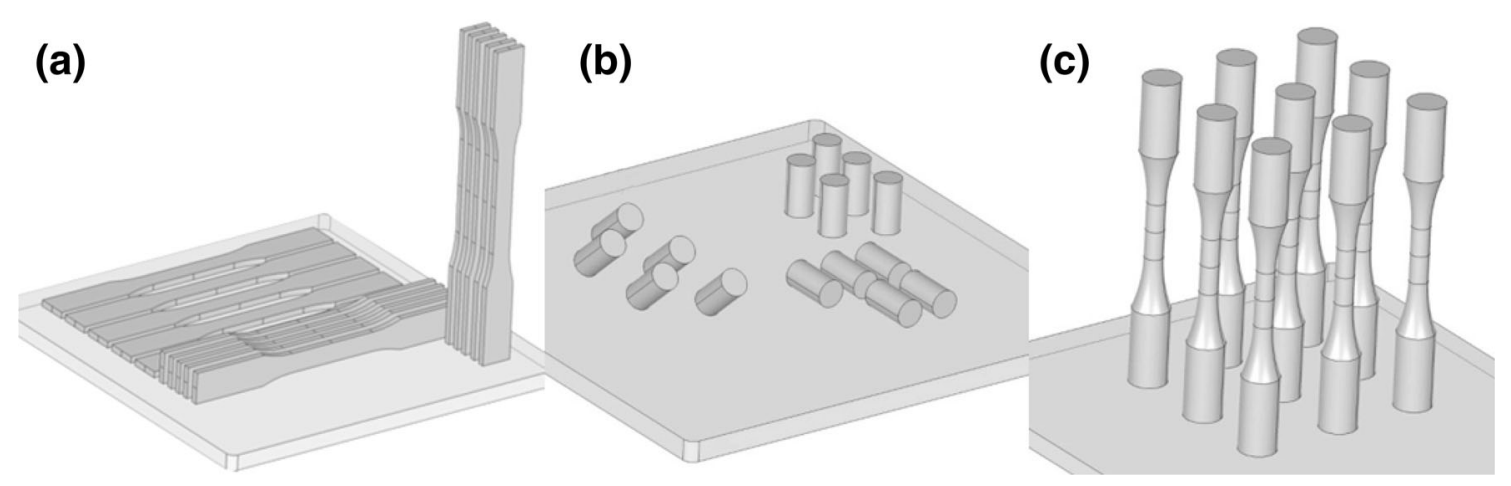

Fig. 3 Building orientation of a tensile, b compression, and $\mathbf{c}$ fatigue test samples located on build plate $($ sizing $250 \times 250 \mathrm{~mm}$ )

Fig. 4 Manufactured LV demonstrator parts: a AISI $316 \mathrm{~L}$ Tripod bracket; b Ti64 Flexible guide; and c AlSi12 Connector support
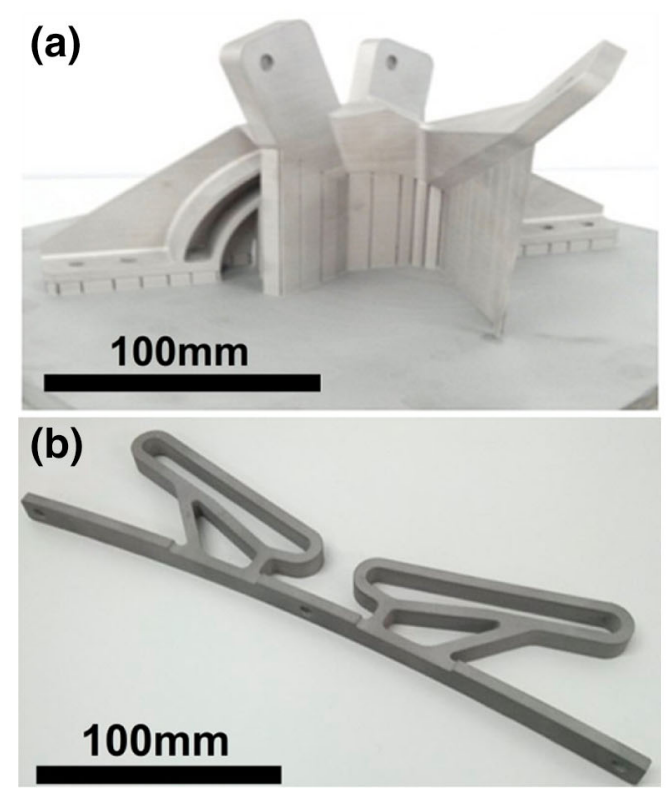

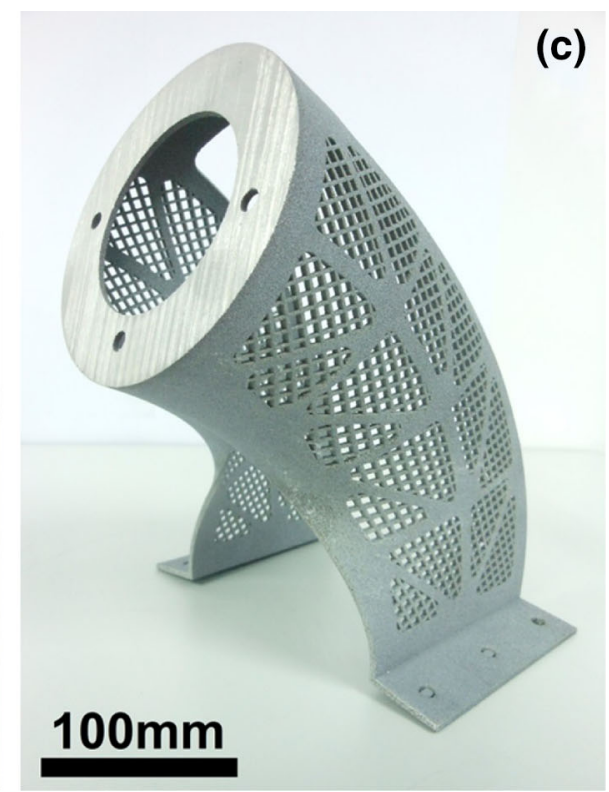

processing technology was the one that showed the best performance according the required criteria, applicability, and cost.

\subsection{Demonstrator NDI evaluation}

Different demonstrator components have been manufactured in different alloys to obtain a preliminary study about the fabrication feasibility using SLM technology (Fig. 4). Quality control and verification have been performed and reported on covering surface roughness measurement, dimensional analysis, and non-destructive evaluation.

The different evaluation and verification processes for the demonstrators executed during the testing campaign were surface roughness analysis (in accordance with ASTM B946-11), dimensional evaluation by means of computed tomography, eddy-current inspection executed according to the ECE standard I + D-E-140, and porosity analysis performed on $1 \mathrm{~cm}^{3}$ density cube samples (manufactured with high-density parameters and together with the demonstrators) by computed tomography.

After inspection, no voids within the tomographic inspection resolution were observed for the different parts. According to the characteristics of the system, the inspection resolution for pore detection in the targeted parts has been defined as a volume of eight times the voxel size: $6.86 \times$ $10^{-3} \mathrm{~mm}^{3}$ for the main beam component, $2.75 \times 10^{-3} \mathrm{~mm}^{3}$ for all guides, and $0.73 \times 10^{-3} \mathrm{~mm}^{3}$ for the pyro support and key latch (the voxel size of the inspections was 95, 70, and $45 \mu \mathrm{m}$, respectively). For the case of cracks, the minimum defect thickness detectability has been defined as half of the voxel size. According to FEM analysis, pores below $15 \times 10^{-3}$ and $1.5 \times 10^{-3} \mathrm{~mm}^{3}$ are not relevant in the critical components, such as the key latch and main beam, respectively, and much larger on the functional elements with respect to the inspection resolution. 
Fig. 5 NDI inspection of LV demonstrator parts. (a) threedimensional representation with actual-to-nominal analysis.

(b) cross section for flaw analysis in connector support demonstrator. (c) Actual-tonominal dimension study in flexible guide demonstrator

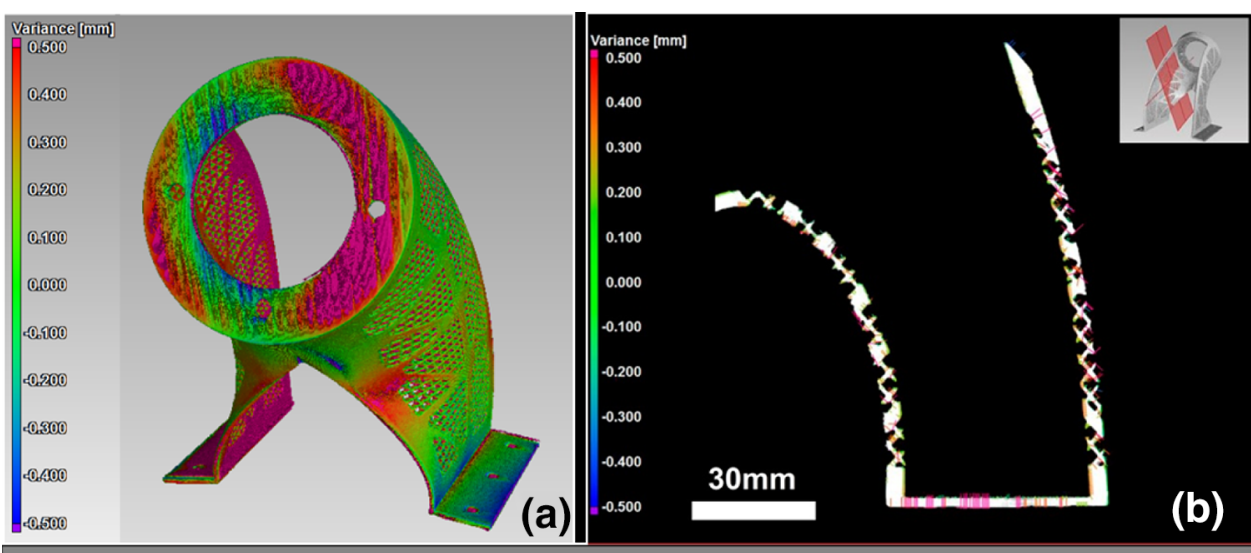

(c)

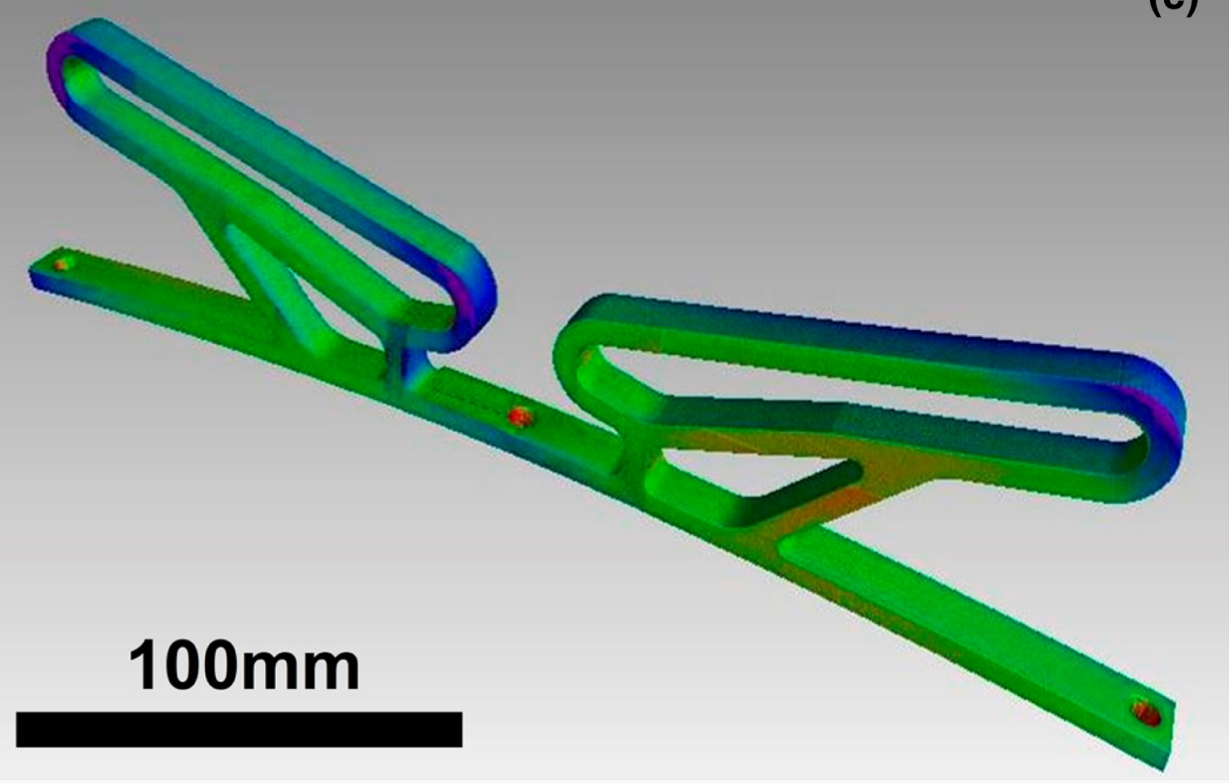

Tomographic inspections include the detection of any flaw along the cross section and 3D representation, and performing actual-to-nominal comparison analysis. Examples of the obtained results can be observed in Fig. 5 . Regarding the dimensional analysis, $>70 \%$ of the surfaces have been between $\pm 0.30 \mathrm{~mm}$.

\subsection{Feasibility analysis for eddy-current and tomographic inspections for flaw detection}

A reference sample has been designed and manufactured (Fig. 6a, b) by SLM to stablish the detectability limitations of eddy-current technology against tomographic evaluation. Several defects ("platelet-like voids" for simulating lack of fusion within the material) have been designed within the part, with thickness from 0.01 to $1 \mathrm{~mm}$. Those have been positioned open to the surface and from 0.5 to $3.0 \mathrm{~mm}$ depth (see Table 1 for details).
For the case of computed tomography (Fig. 6c, d), all indications have been detected. On the other hand, eddycurrent technology has been capable of detecting most of surface defects and with thicknesses larger than $0.1 \mathrm{~mm}$. It must be mentioned that the technique was not able to detect the artificial defects on the as-manufactured surface, requiring a post-machining step.

\section{Demonstrator: design, manufacturing, verification, tests, and technology evaluation}

Finally, a breadboard has been defined for fabrication: a full-scale low shock clamp band separation system, currently in use for AR5. The last has an important grow potential in terms of performance and a relevant number of components susceptible to be made with ALM technology. The parts of this system have severe weight constrains and 
(a)

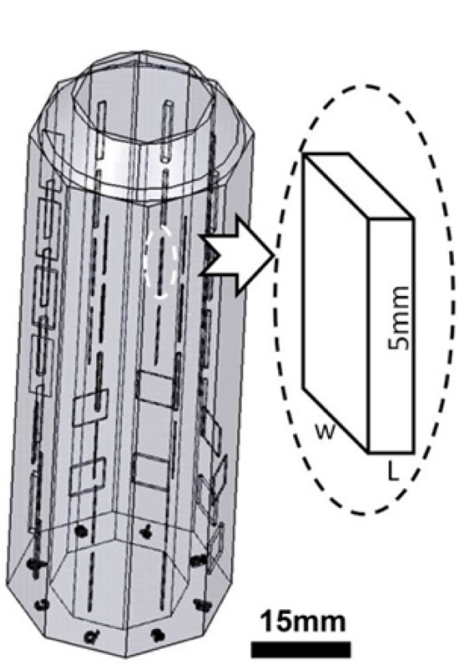

(b)

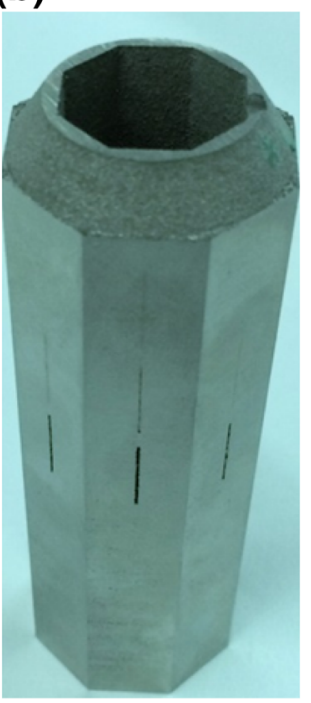

(c)

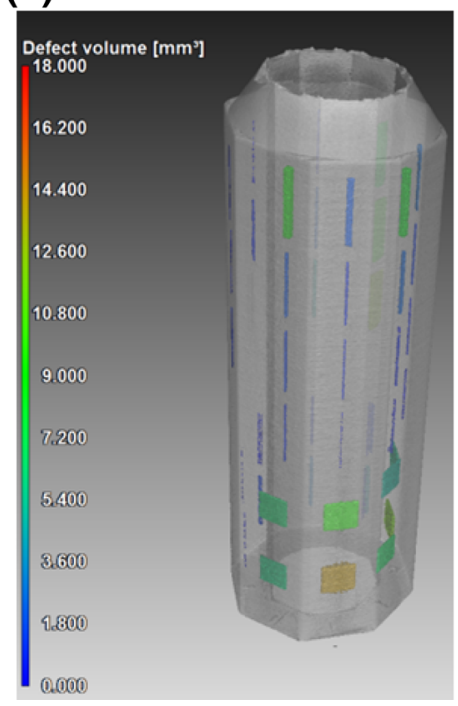

(d)

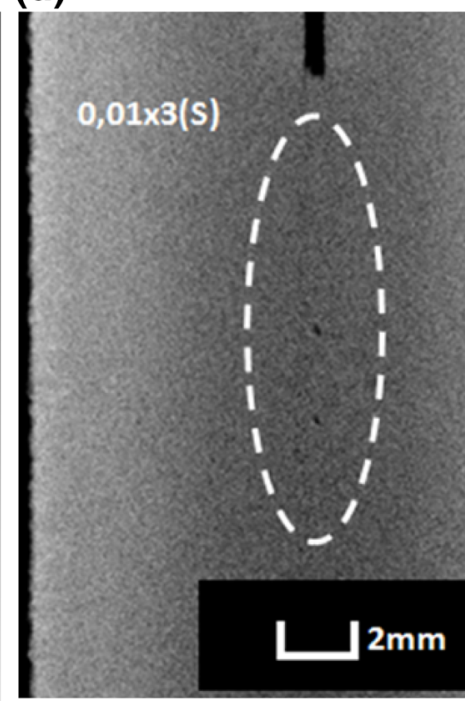

Fig. 6 a Schematic design and $\mathbf{b}$ view of the developed reference sample for the analysis of EC and XCT detectability; c 3D representation of artificial defects (flaws colored according to their volume; and $\mathbf{d}$ cross section showing created porosity for $0.01 \mathrm{~mm}$ thickness defects: indication $0.01 \times 3(S)$ (color figure online)

Table 1 Detail of artificial defect aspect (thickness, wide, and length) on NDT standard

\begin{tabular}{|c|c|c|c|c|c|c|}
\hline \multirow{2}{*}{$\begin{array}{l}\text { Part face } \\
\text { A }\end{array}$} & \multicolumn{6}{|c|}{$\begin{array}{l}\text { Thickness } \times \text { wide }(\text { depth })(\mathrm{mm}) \\
* \mathrm{~S}=\text { on the surface; } \mathrm{P} 0.5=0.5 \mathrm{~mm} ; \mathrm{P} 1=1 \mathrm{~mm} ; \mathrm{P} 2=2 \mathrm{~mm} \text {; and } \mathrm{P} 3=3 \mathrm{~mm} \\
\text { Length }=5 \mathrm{~mm} \text { for all defects }\end{array}$} \\
\hline & $0.1 \times 0.2(\mathrm{~S})$ & $0.2 \times 0.2(\mathrm{~S})$ & $0.5 \times 0.2(\mathrm{~S})$ & $0.05 \times 0.2(\mathrm{~S})$ & $0.1 \times 0.2(\mathrm{P} 1)$ & $0.1 \times 0.5(\mathrm{P} 1)$ \\
\hline $\mathrm{B}$ & $0.1 \times 0.5(S)$ & $0.2 \times 0.5(\mathrm{~S})$ & $0.5 \times 0.5(\mathrm{~S})$ & $0.05 \times 0.5(\mathrm{~S})$ & $0.2 \times 0.2(\mathrm{P} 1)$ & $0.2 \times 0.5(\mathrm{P} 1)$ \\
\hline $\mathrm{C}$ & $0.1 \times 1.0(S)$ & $0.2 \times 1.0(\mathrm{~S})$ & $0.5 \times 1.0(S)$ & $0,05 \times 1.0(\mathrm{~S})$ & $0.5 \times 0.2(\mathrm{P} 1)$ & $0.5 \times 0.5(\mathrm{P} 1)$ \\
\hline $\mathrm{D}$ & $0.1 \times 3.0(S)$ & $0.2 \times 3.0(\mathrm{~S})$ & $0.5 \times 3.0(S)$ & $0.01 \times 3.0(\mathrm{~S})$ & $1.0 \times 0.2(\mathrm{P} 1)$ & $1.0 \times 1.0(\mathrm{P} 1)$ \\
\hline $\mathrm{E}$ & $1.0 \times 0.2(\mathrm{P} 1)$ & $0.5 \times 0.2(\mathrm{P} 1)$ & $0.2 \times 0.2(\mathrm{P} 1)$ & $0.1 \times 0.2(\mathrm{P} 1)$ & $5.0 \times 0.2(\mathrm{P} 3)$ & $5.0 \times 0.4(\mathrm{P} 3)$ \\
\hline $\mathrm{F}$ & $1.0 \times 1.0(\mathrm{P} 2)$ & $0.5 \times 0.5(\mathrm{P} 2)$ & $0.2 \times 0.5(\mathrm{P} 2)$ & $0.1 \times 0.5(\mathrm{P} 2)$ & $5.0 \times 0.2(\mathrm{P} 2)$ & $5.0 \times 0.4(\mathrm{P} 2)$ \\
\hline G & $1.0 \times 0.2(\mathrm{P} 3)$ & $0.5 \times 0.2(\mathrm{P} 3)$ & $0.2 \times 0.2(\mathrm{P} 3)$ & $0.1 \times 0.2(\mathrm{P} 3)$ & $5.0 \times 0.2(\mathrm{P} 1)$ & $5.0 \times 0.4(\mathrm{P} 1)$ \\
\hline $\mathrm{H}$ & $1.0 \times 1.0(\mathrm{P} 3)$ & $0.5 \times 0.5(\mathrm{P} 3)$ & $0.2 \times 0.5(\mathrm{P} 3)$ & $0.1 \times 0.5(\mathrm{P} 3)$ & $5.0 \times 0.2(\mathrm{P} 0.5)$ & $5.0 \times 0.4(\mathrm{P} 0.5)$ \\
\hline
\end{tabular}

All defects detected by computed tomography. In bold, only defects detected by eddy-current inspection

high-critical requirements in terms of stiffness, strength, tolerances, and critical surface finishing. All the above aspects have been considered for its selection.

The separation system, named LPSS937* and designed for AR5 (Fig. 7), is used as clamping device to transmit the loads between the launcher and the spacecraft (S/C) during the flight. In addition, the opening of the band allows the release and jettisoning of the S/C.

A complete design, manufacturing and test processes have been performed in the breadboard, with functional and loading requirements equivalent to the flight unit. It has been substituted some components of the LPSS937* by others performed by AM, to check the feasibility of this new design with similar shape and mass, but partially manufactured by ALM process.
Nine (9) different components of the clamp band were identified as potential candidates to be manufactured by AM technology (and depicted in Table 2). Related to its size and other manufacturing issues, the main beam component has been manufactured by EBM technology. The rest of the parts were fabricated using SLM processing. According to the conventional specifications, the selected material for the fabrication of the parts was Ti64.

\subsection{Manufacturing issues}

Once the breadboard design was established, the items were manufactured by AM. The performance objectives to be accomplished during the manufacturing and testing are presented in Table 3: 


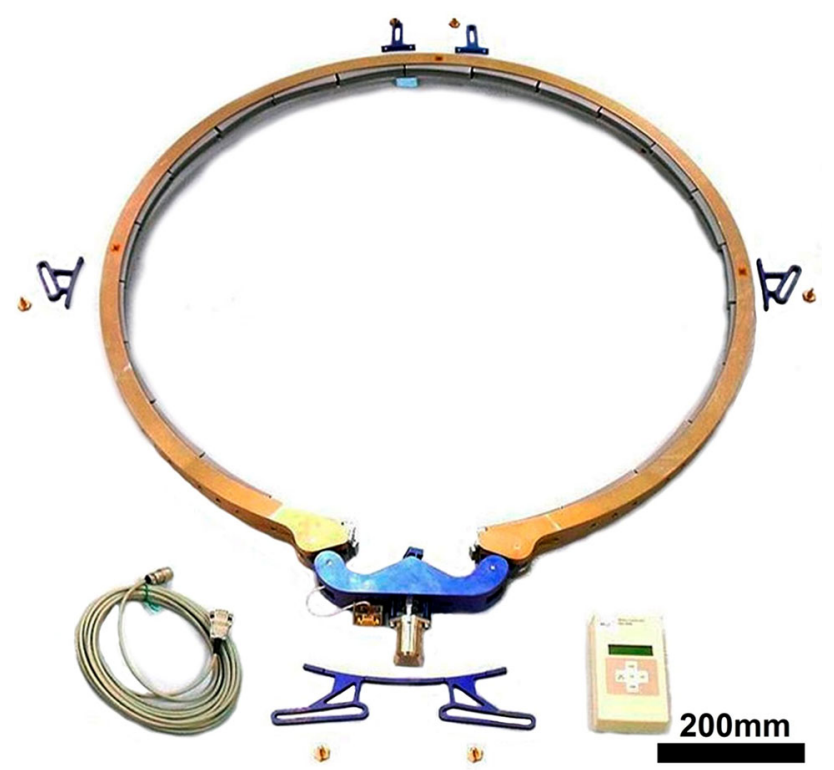

Fig. 7 View of Ariane5 LPSS 937* (diameter of the system $=937 \mathrm{~mm}$ )

Before manufacturing, the previous design stage, setup preparation and building strategies were established, for each part from the breadboard design.

According to the part performance and due the maximum manufacturing volume allowed by the manufacturing system, the "main beam flexible guide" component has been manufactured into two different parts along its axis of symmetry.

\subsection{Breadboard results}

To optimize the manufacturing process according to the breadboard parts positioning and orientation, the different items were carried out into five different building plates. On each manufacturing plate, tensile tests specimens were manufactured simultaneously for indirect process monitoring issues, as well as reference blocks for microstructural characterization and void content analysis. Examples of building plates are presented in Fig. 8 .

\subsection{Breadboard NDI inspection}

Evaluation and verification processes have been carried out for the different breadboard parts. X-ray computed tomography (XCT) porosity analysis was performed on density check samples with high-density parameters on the manufacturing plate. The dimensional analysis was performed by XCT, and the surface roughness was approached in accordance with ASTM B946-11.

During the porosity analysis, no voids were found within inspection resolution of each part (between 45 and $95 \mu \mathrm{m}$ ). XCT was as well applied for check density samples

Table 2 Breadboard ALM design elements

\begin{tabular}{|c|c|c|c|c|}
\hline Part & View & Material & Quantity & AM technology \\
\hline Main beam & & Ti64 & 1 & EBM \\
\hline Main beam/flexible guide & & Ti64 annealed & 2 items/part 1 and 2 & SLM \\
\hline Key latch & & Ti64 annealed & 1 & SLM \\
\hline $45^{\circ}$ flexible guide & & Ti64 annealed & 2 (right and left) & SLM \\
\hline Pyro support & & Ti64 annealed & 1 & SLM \\
\hline $180^{\circ}$ flexible guide & & Ti64 annealed & 2 (right and left) & SLM \\
\hline
\end{tabular}


Table 3 Performance objectives

\begin{tabular}{ll}
\hline Breadboard & Performance objectives \\
\hline Manufacturing & Complete part and features manufactured according to the CAD, orientation and supports design \\
NDE flaw detection & Porosity characterization by X-ray-computed tomography on samples checks at different height levels with \\
the following target: & Void content below $1 \%$ \\
& No porosity gradient along building direction \\
& Crack characterization by tomography \\
& Non I/F surfaces Successful nominal to actual analysis on manufactured parts with deviation on below \\
& \pm 0.5 mm \\
PDE dimensional analysis & Part thickness above nominal value \\
& Non I/F surfaces $<$ finishing below to $10 \mu$ m after sand blasting \\
Surface characterization and & I/F surfaces $<$ finishing according to the specification \\
engineering & Static/gap test tensioning of the LPSS until 45kN. No failure of the components at load level \\
Testing campaign & Separation tests complete system released, without significant disturbances (lateral impulse) and failure \\
\hline
\end{tabular}
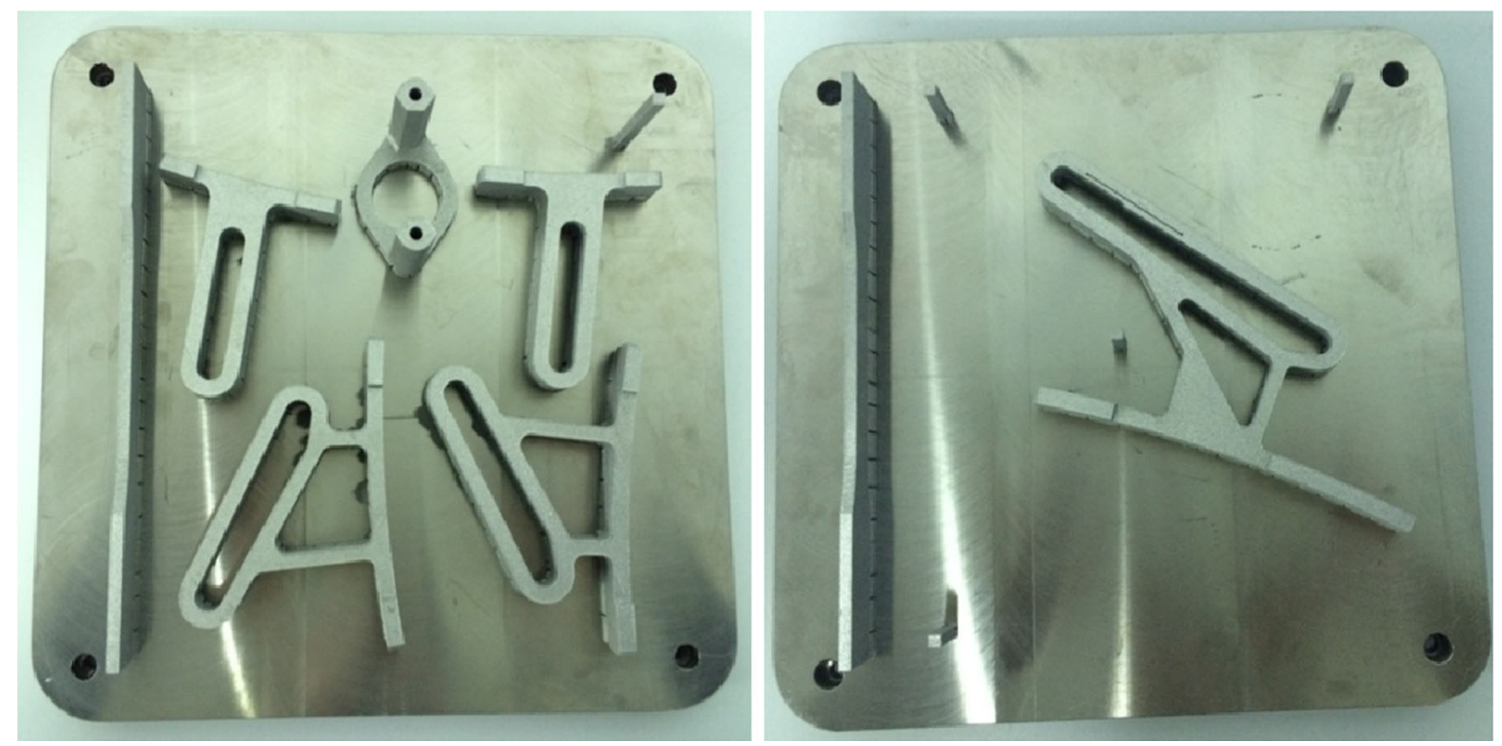

Fig. 8 Views of two building plates (sizing $250 \times 250 \mathrm{~mm}$, each), including parts and specimens for verification

manufactured the same manufacturing plates ( 5 in total). The overall void content was quantified between 0.05 and $0.47 \%$ (below the performance objective of $1 \%$ ). Other aspect were analysed, such as the distribution of porous size (including the contribution percentages to the overall porosity of the different porous sizes), pore morphology, and sphericity.

The surface roughness analysis was performed measuring the surface roughness at different locations (including different analysis orientations according to fabrication direction). Roughness values between 6.9 and 12.1, and 8.0 and $11.4 \mathrm{Ra}(\mu \mathrm{m})$ were recorded for $Z$ and $X Y$ directions, respectively.
Nominal/actual analysis 3D views of the different components were performed, comparing nominal and resultant dimensions, after tomographic reconstruction and CAD design model. The data set under examination has been color-coded according to deviations vs. nominal CAD. For all components, $>85 \%$ of the surfaces have been evaluated between $\pm 0.30 \mathrm{~mm}$.

\subsection{Full system acceptance tests}

The main objective of the tests is to demonstrate the strength and functionality of the additive manufactured LPSS 937*. Figure 9 shows views of the conventional and 

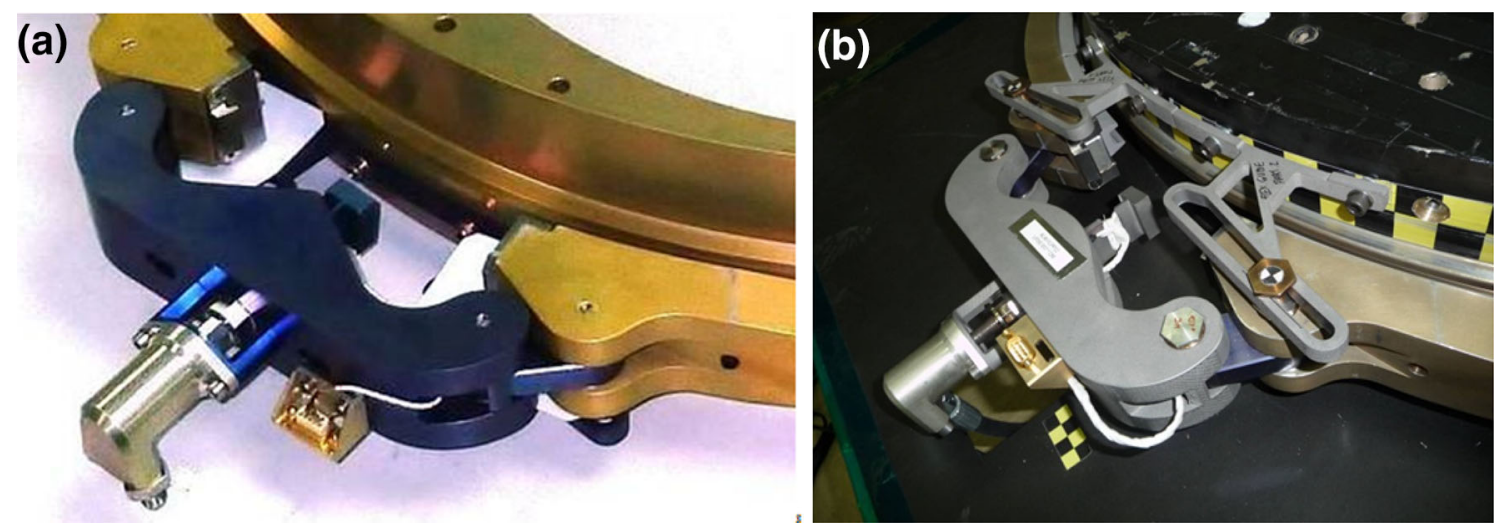

Fig. 9 LPSS 937*. Flight closure mechanism (a) ALM demonstrator (b)
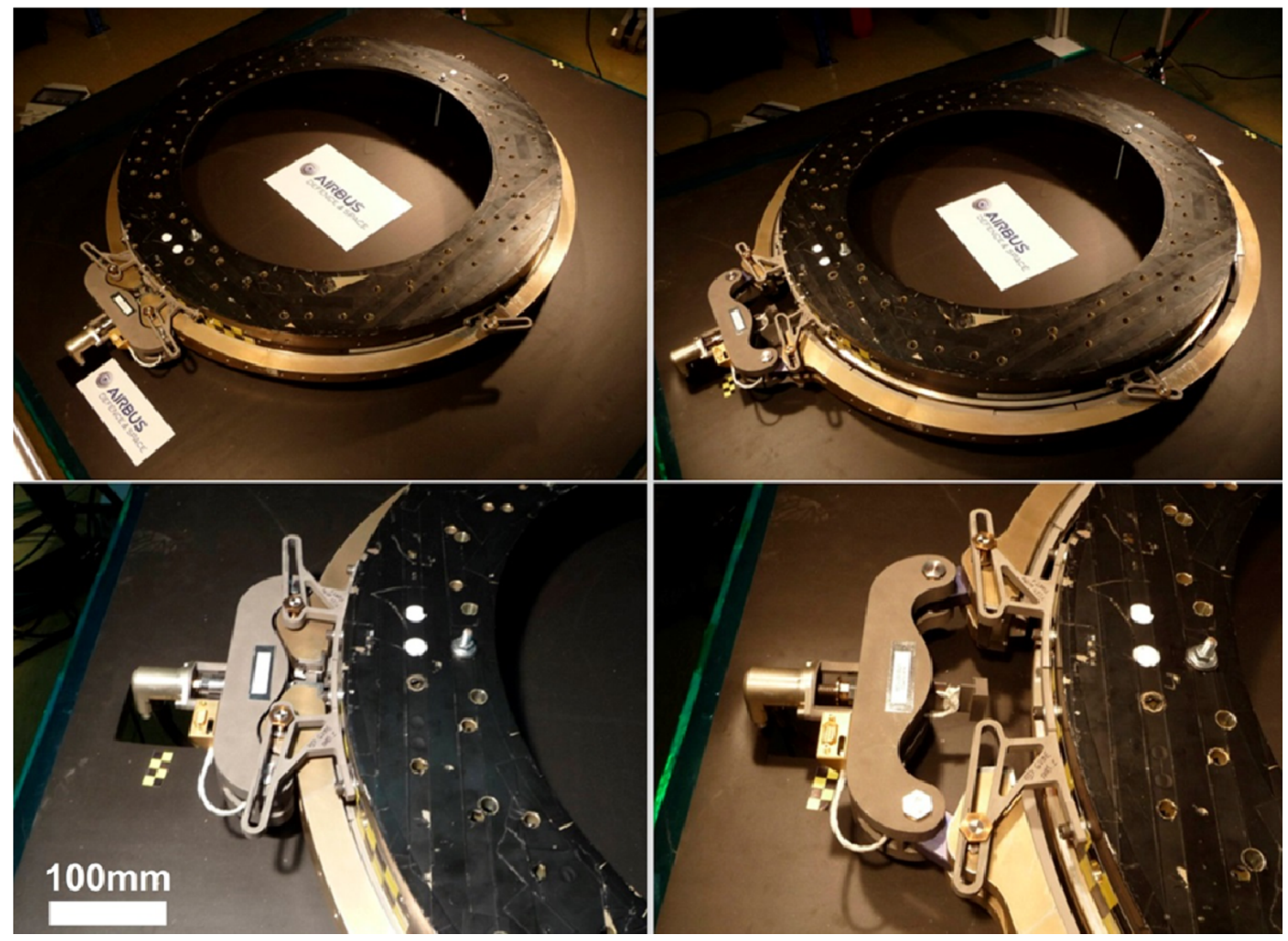

Fig. 10 ALM demonstrator test sequence: closed (left) open (right)

AM manufactured system, including close and open configurations as well.

The clamp band system has been subjected to a tension and release tests in similar qualification conditions that the current version of the system. The acceptance campaign was performed to demonstrate both its ability to be tensioned up to the maximum flight tension value $(45 \mathrm{kN})$ and its functional performance through several release tests. To demonstrate the system functionality, three band tensioning operations were performed in the following order: minimum $30 \mathrm{kN}$, maximum $45 \mathrm{Kn}$, and nominal $40 \mathrm{kN}$ (see in Fig. 10 the test rig and testing sequence).

Finally, no degradation/malfunction was observed during the inspection made after the acceptance campaign. The tests were successfully executed, demonstrating the AM pieces good strength and correct functionality even for a tension value higher than the specified nominal one. A view of the final demonstrators can be observed in Fig. 11. 


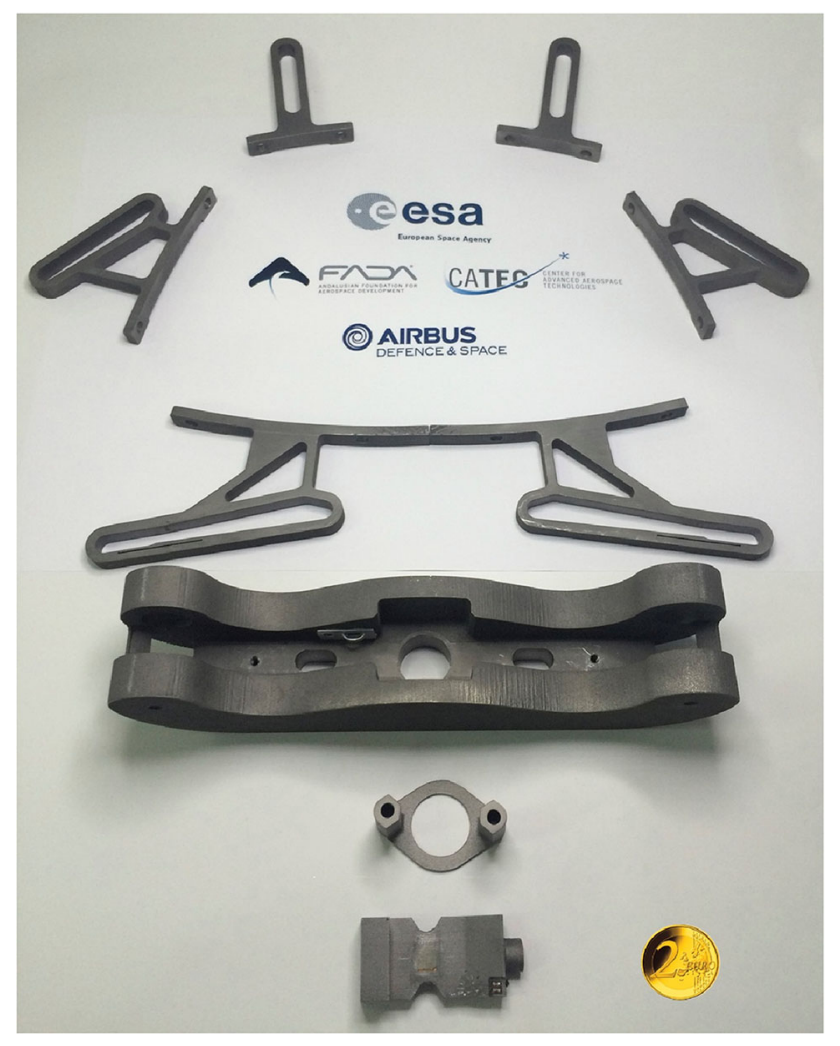

Fig. 11 LPSS parts manufactured and tested

\section{Conclusions}

A deep study of AM applicability for different elements of launcher mechanical systems (excluding the big field of propulsion) has been performed, identifying main drivers and limitations of existing technologies and materials. The main conclusions of the study are the following:

Current maximum dimensions reachable with most of the available AM technologies recommend concentrating the short-term efforts in small parts, such as brackets and supports.

Aluminium-based elements, where the main driver for the design is the stiffness (more than strength), can benefit from AM, especially if a topology optimization exercise is implemented. An example of this is the payload adapter connector bracket for Vega Launcher [3].

There is a large application field for elements made from Titanium alloys. There, a distinction must be performed between already existing Titanium brackets from those including complex geometries/"bionic" geometries. The last can be designed and manufactured using topology optimization procedures, leading into mass reductions and the cost benefit (reduction of manufacturing time against conventional machining) in such high strength alloy. Furthermore, aluminium brackets can be modified into Titanium ones, where topology optimization can even obtain mass and cost gains, and overcoming the problem of the not commercially available high strength aluminium alloy for AM.

Process control-including inspection and qualification approaches-is the key aspect for future launcher applications, where a serial production is expected. Computed tomography shows a great potential for its applicability for non-destructive evaluation. Due to the inspection cost, the manufacturing of test articles within each fabrication batch must be considered to check indirectly the build process. At the same time, online monitoring strategies are being implemented within AM systems [9], which can help in assuring product quality and decrease inspection costs.

Acknowledgments The authors would like to thank the European Space Agency for the funding of the ALM project (Additive Layer Manufacturing-ALM; Future Launchers Preparatory Programme; FLPP 2.2, M\&S CUSS). The authors would like to thank Dr. Andreas Tesch (ESA project responsible), Dr. Laurent Pambaguian and Dr. Tommaso Guidini for their support.

\section{References}

1. Tomlin M, Meyer J (2011) Topology optimization of an additive layer manufactured (ALM) aerospace part. In: The 7th Altair CAE Technology Conference, pp 1-9

2. Emmelmann C, Sander P, Kranz J, Wycisk E (2011) Laser additive manufacturing and bionics: redefining lightweight design. Phys Procedia 12A:364-368

3. Lasagni F, Zorrilla A, Periñán, Tudela S, Vilanova J (2014) On the investigation of processing parameters and NDT inspection on additive manufacturing materials for future launchers. In: Workshop on additive manufacturing for space applications, ESA-ESTEC, Noordwijk, The Netherlands

4. Vilanova J, Romera P, Lasagni F, Zorrilla A, Periñán A (2014) Additive layer manufacturing for launcher's applications. In: Proceedings 13th European conference on spacecraft structures, materials \& environmental testing, 1-4 April, Braunschweig, Germany

5. ASTM F2792-12a (2015) Standard terminology for additive manufacturing technologies

6. Wohlers Report 2015 (2015) 3D Printing and additive manufacturing state of the industry. Wohlers Associates, Fort Collins

7. Box GEP, Hunter JS, Hunter WG (2005) Statics for experiments: desing, innovation and discovery. Wiley, Hoboken

8. Lasagni F, Lasagni A, Marks E, Holazapfel C, Mücklich F, Degischer HP (2007) Three dimensional characterization of as-cast and solution treated AlSi12(Sr) alloys by high-resolution FIB tomography. Acta Materialia 55:3875-3882

9. Jordan C (2014) EOS and plasmo join forces in the field of online process monitoring for Additive Serial Manufacturing. http://www. eos.info/eos_plasmo_online_process_monitoring_for_additive_man ufacturing. Accessed Aug 2004 